\title{
GMRT survey of Cygnus OB2 region
}

\author{
C. H. Ishwara-Chandra ${ }^{1 *}$, Paula Benaglia ${ }^{2}$, Michaël De Becker ${ }^{3},{\text { Anandmayee } \text { Tej }^{4}}^{4}$ \\ ${ }^{1}$ NCRA-TIFR, Post Bag No. 3., Ganeshkhind, Pune - 411007, India \\ ${ }^{2}$ Instituto Argentino de Radioastronomia, CONICET \& CICPBA, CC5 (1897), \\ Villa Elisa, Prov. de Buenos Aires, Argentina \\ ${ }^{3}$ Space sciences, Technologies and Astrophysics Research (STAR) Institute, University of Liége, \\ Quartier Agora, 19c, Allée du 6 Aôut, B5c, B-4000 Sart Tilman, Belgium \\ ${ }^{4}$ Indian Institute Of Space Science And Technology, Thiruvananthapuram 695 547, Kerala, India
}

\begin{abstract}
A good fraction of very high energy (VHE; $>100 \mathrm{GeV}$ ) sources do not have confirmed counterparts at any of the other branches of electromagnetc spectrum. In the extra-galactic sky, most of the VHE sources with counter-parts are blazars, a sub-class of active galactic nuclei. However, VHE sources with identified counterparts in our Galaxy do have a wide range of categories, ranging such as supernovae remnants, colliding wind binaries, pulsar wind nebulae, micro-quasars, etc. The VHE emission and radio emission due to synchrotron process do occur from the same population of relativistic electrons in several type of sources, so the radio band is the first band to search for possible counterparts to unidentified VHE sources. In addition, the radio spectral index also gives important constraints on compactness and energetics of the electron population. In this work we present a two-frequency radio survey, at frequencies $325 \mathrm{MHz}$ and $610 \mathrm{MHz}$, of the Cygnus OB2 region with the Giant Metrewave Radio Telescope (GMRT) in order to address several astrophysical problems. The survey covers a wide area ( $\sim 5$ square degrees) at sub-mJy sensitivity which helps to discover faint radio sources to several objects for the first time. Also this is the first deep wide area radio survey of the region below $1.4 \mathrm{GHz}$, and the low radio frequency will preferentially pickup more non-thermal radio sources as compared to high radio frequencies. Here, we present the early results from this survey.
\end{abstract}

Keywords: - Galaxy: Open Clusters and Associations - Individual: Cygnus OB2, 8, 9 - Radio Continuum: General - Surveys

\section{Introduction}

A significant fraction of the very high energy (VHE; $>100 \mathrm{GeV}$ ) sources remain unidentified, in the sense that there is no counterpart in any other wavelength (e.g. Massaro et al. 2013). While nearly all of the identified extra-galactic VHE sources are active galactic nuclei (AGN) such as blazars, the possible counterparts for such sources within our galaxy can be many. The investigation of these counterparts relies on the exploration of Galactic environments known to be able to participate in non-thermal processes. Supernova remnants and pulsar wind nebulae are known to be efficient particle accelerators and noticeable synchrotron radio sources (e.g. Dubner \& Giacani 2015, Reynolds

${ }^{*}$ E-mail: ishwar@ncra.tifr.res.in 
et al. 2012). Microquasars, and especially the interaction between the high energy protons which are accelerated at their jet termination shock can produce secondary particles that radiate via synchrotron process (e.g. Bosch-Ramon et al. 2005). Colliding wind binaries made of massive stars are also known to be particle accelerators and non-thermal emitters (De Becker \& Raucq 2013, De Becker et al. 2017).

Some of the unidentified VHE sources are also very extended, which makes the search for counterparts difficult. If there are unidentified VHE sources in the Galaxy, the search for their counterparts should have a wider search matrix to cover all possible options. One of the main limitations to search for counterparts is the large position error. There can be several sources within the large error box which makes it difficult to uniquely identify the counterpart. In addition, one caution that positional coincidence does not necessarily mean physical connection, and deeper multi-wavelength investigations are needed to fully address the issue of source identification.

One of the most promising spectral domain to look for the counterpart of VHE sources is thus the radio domain. If the VHE emission is due to inverse Compton emission then the same relativistic electrons will also give rise to radio emission in the presence of magnetic field via the synchrotron process. Due to this, there will be spatial coincidence of VHE and radio emission and also possibility of correlated variability between VHE and radio emission.

Here we describe preliminary results on our work on low radio frequency survey of Cygnus OB2 region using the Giant Metrewave Radio Telescope (GMRT). In Section 2, we describe the telescope and in Section 3 we give details of the survey. Initial results are presented in Section 4, while future outlook and concluding remarks are given in Section 5.

\section{The Giant Metrewave Radio Telescope}

The GMRT consists of thirty $45 \mathrm{~m}$ diameter antennas (Figure 1) spread over a $25 \mathrm{~km}$ region. Half the antennas are in a compact, quasi randomly distributed array with a diameter of about $1 \mathrm{~km}$. The remaining antennas are on three arms of length $\sim 14 \mathrm{~km}$ (NorthWest, NorthEast and South) with 5 or 6 antennas on each arm. The longest baseline is about $25 \mathrm{~km}$ and the shortest is about $100 \mathrm{~m}$ without foreshortening.

The telescope $\left(\right.$ latitude $=19.1^{\circ} \mathrm{N}$, longitude $=74.05^{\circ} \mathrm{E}$ ) is located near Khodad village, which is about $80 \mathrm{~km}$ north of Pune. The GMRT antennas are $45 \mathrm{~m}$ alt-azimuth mounted parabolic primefocus dishes. The reflecting surface is formed by wire mesh and the efficiency of the antennas varies from $60 \%$ to $40 \%$, from the lowest to the highest frequency. There is a rotating turret at the focus on which the different feeds are mounted. The feeds presently available are the band-5 (1000-1460 MHz), band-4 (550-850 MHz), band-3 (250-500 MHz) and band-2 (130-260 MHz) ones. The broadband RF signal is brought directly to the receiver room, where it is converted to baseband signals with a maximum bandwidth of $400 \mathrm{MHz}$. This is processed with a new digital back-end system (correlator + beamformer + pulsar receiver), that can handle the full $400 \mathrm{MHz}$ bandwidth. The spectral resolution of the correlator can be 2048, 4096, 8192 and 16392 channels. Some useful system parameters are given in Table 1. 


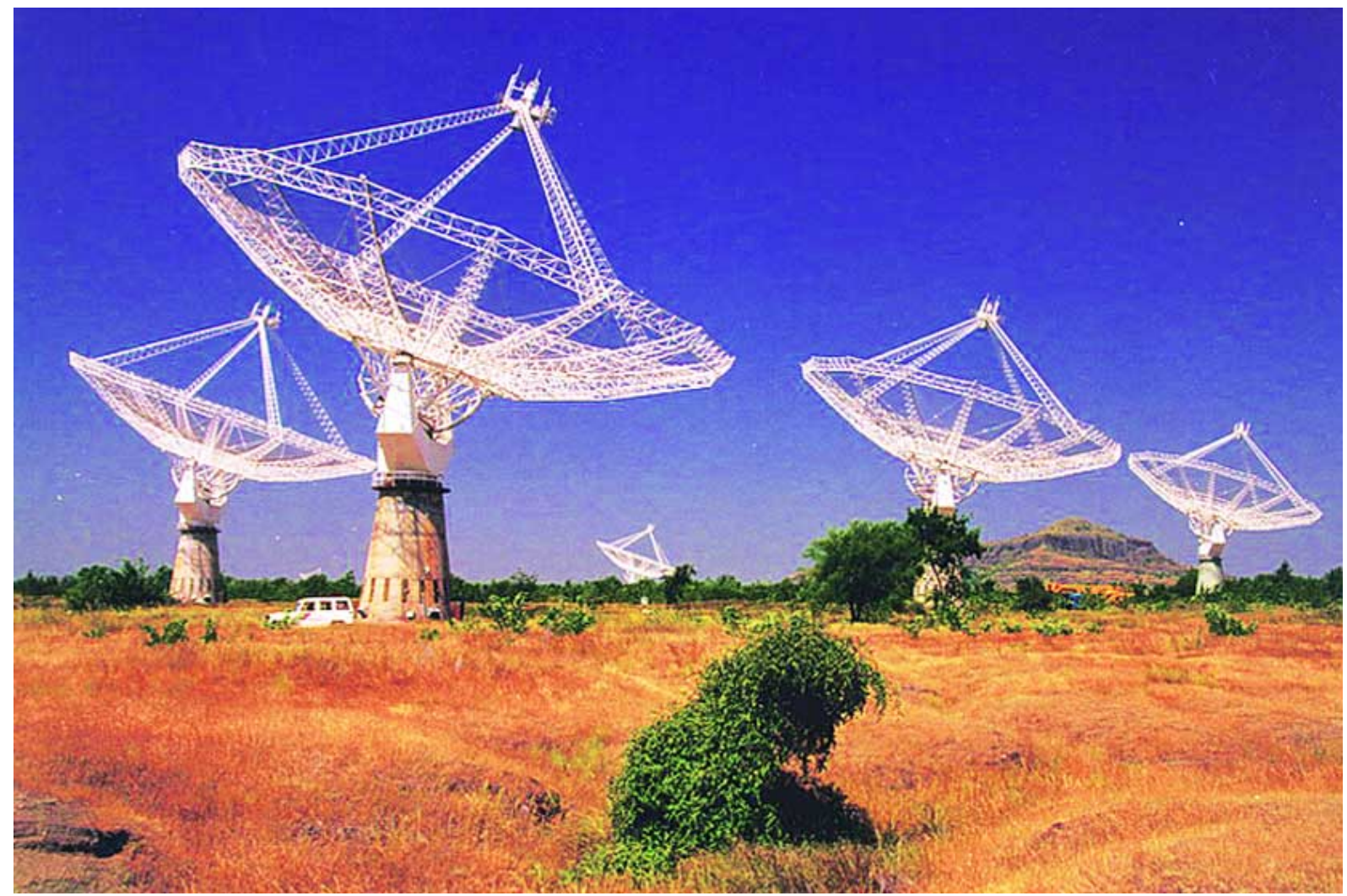

Figure 1: A few antennas of the GMRT array.

Table 1: System Parameters of the upgraded GMRT (uGMRT).

\begin{tabular}{lllll}
\hline \hline Band & Gain & Total Tsys (K) & $\begin{array}{l}\mathrm{rms}^{\$} \\
\left(\mu \mathrm{Jy}_{\text {beam }}-1\right)\end{array}$ & $\begin{array}{l}\text { Synthesized Beam } \\
\text { (arcsec, band center) }\end{array}$ \\
\hline band-2 (130 to $260 \mathrm{MHz})$ & 0.33 & 760 to 240 & 190 & 17.3 \\
band-3 (250 to $500 \mathrm{MHz})$ & 0.38 & 165 to 100 & 50 & 8.3 \\
band-4 (550 to $850 \mathrm{MHz})$ & 0.35 & 105 to $100 \%$ & 40 & 4.3 \\
band-5 (1000 to $1460 \mathrm{MHz})$ & $0.28-0.22$ & 80 to 75 & 45 & 2.3 \\
\hline \hline
\end{tabular}

\%: This is for $800 \mathrm{MHz}$, at $825 \mathrm{MHz}, \mathrm{T}_{\text {sys }}$ is $133 \mathrm{~K}$, and $\mathrm{T}_{\text {sys }}$ increases beyond.

$\$$ : rms is determined assuming 30 antennas, 10 min integration and $100 \mathrm{MHz}$ bandwidth.

\section{GMRT Survey of Cygnus OB2 region}

The Cygnus region in our galaxy is rich with many high-energy sources. It is an active star-forming region relatively close-by, among the richest and crowded in the Galaxy (see Reipurth \& Schneider 2008 for a review). The Cygnus region houses nine OB associations together with bright open clusters, represented and labeled in Fig. 2 (from Mahy et al. 2013). The high absorption in the line of sight, however, prevents to accurate mapping the stellar population. Cyg OB2 is one of the youngest groups, with a hundred O stars and thousands of B stars (e.g. Knödlseder et al. 2003). Cyg OB1, 8 and 9 harbor about a hundred hot stars. There are also large number of unidentified VHE sources in this region.

We have conducted a wide area low frequency radio survey of Cygnus region with GMRT at 325 $\mathrm{MHz}$ and $610 \mathrm{MHz}$ with several science goals in mind. One, to search for counterparts to unidentified VHE sources from the current VHE telescopes and also will form the basis for quick check for 


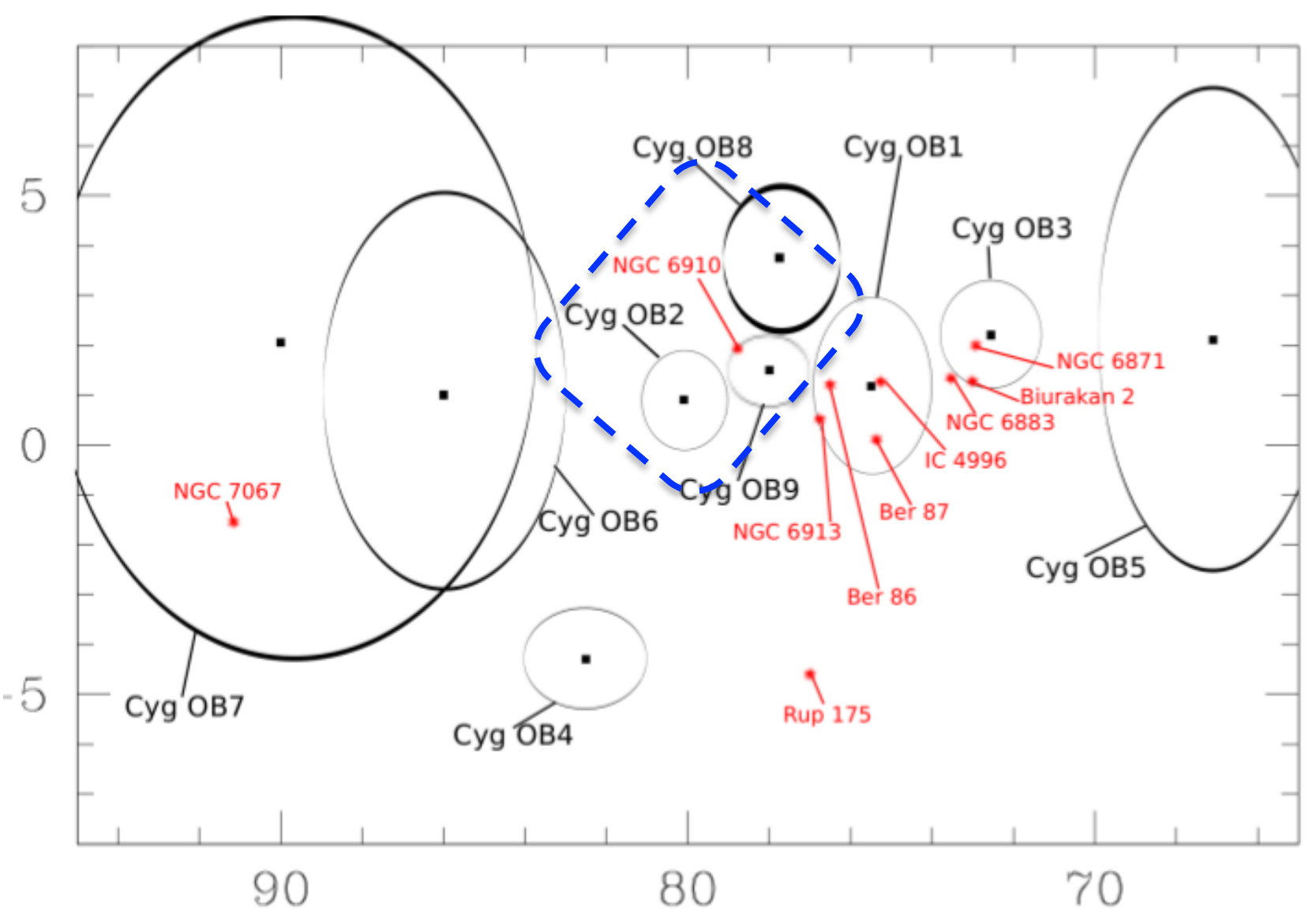

Figure 2: Associations (in black font) and bright clusters (in red font) of the Cygnus Rift, adapted from Mahy et al. (2013) with permission (c) ESO). The dashed blue box marks the region of the survey carried out with the GMRT.

counterparts of upcoming VHE imaging of this region. Two, there are hundreds of massive stars for which the radio counterparts can be searched in a statistically meaningful manner. Third, there will be several other kind of sources like pulsar wind nebulae, colliding wind binaries, etc for which the radio counterparts can be searched. The spectral index from the two frequency observations will be useful to differentiate between thermal and non-thermal process. The resolution of the survey is $<10$ arcseconds which will serve to localise the position accurately as compared to large positional uncertainties in the VHE emission. The sub-mJy sensitivity of the survey at both frequencies is among the deepest radio data of this region.

\subsection{MHz survey}

The $325 \mathrm{MHz}$ survey was carried out with 5 pointings (see Fig. 3). The GMRT field-of-view at this frequency is $\sim 80$ arcmin. In addition, to look for variability, one of the pointings, corresponding to the central area of the association OB2, was observed for two epochs separated by several weeks. The data reduction was performed with the Astronomical Imaging Processing System (AIPS) routines, following the standard procedures. The data was also analysed using the Source Peeling and Atmospheric Modelling (SPAM) pipeline (Intema et al. 2009). The individual images were mosaiced together using mosaic script (Intemma, private communication). The synthesized beam attained was $\sim 10 " \times 10^{\prime \prime}$. The average rms resulted in $\sim 0.5 \mathrm{mJy}^{\prime}$ beam $^{-1}$. This is among the deepest and high resolution survey at this frequency. Since the non-thermal process predicts higher 
flux at low radio frequencies $\left(\mathrm{S}_{\nu} \propto \nu^{\alpha}\right.$, with negative spectral index $\left.\alpha\right)$, we expect more radio sources at low radio frequencies. Also, the contribution of thermal emission to the total radio flux will be less at this frequency, as the latter is characterized by a positive spectral index.

\section{2 $610 \mathrm{MHz}$ survey}

The $610 \mathrm{MHz}$ survey was carried out with $\sim 45$ pointing (see Figure 3, right panel). The central area of Cyg OB2 was observed at two epochs. The $610 \mathrm{MHz}$ band FoV was $\sim 45$ arcmin. The data reduction was performed with AIPS and also using the SPAM pipeline (Intema et al. 2009). The resolution of the image is $\sim 6 " \times 6$ " with an average rms of $0.2 \mathrm{mJy}^{\prime}$ beam $^{-1}$. With this resolution, we can achieve position accuracy of better than 1 arcsecond which will help to uniquely identify counterparts.
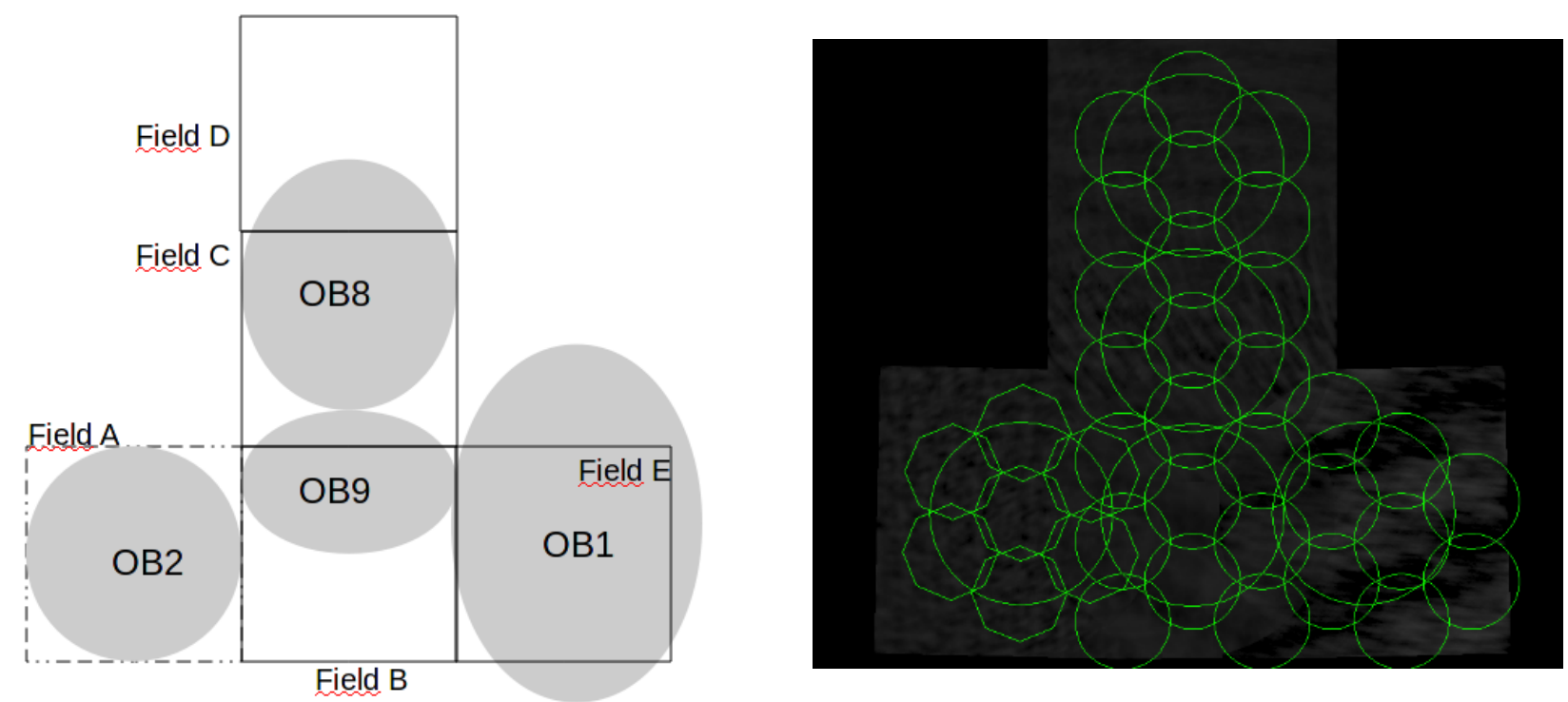

Figure 3: Survey fields in the Cygnus region. Left: observed at $325 \mathrm{MHz}$, with the approximate locations of some associations. Right: observed at $610 \mathrm{MHz}$ (green hexagons) superposed to those at $325 \mathrm{MHz}$ (larger green circles).

\subsection{Individual sources}

The observations at the two bands, with similar angular resolution, will allow to derive spectra index information about a plethora of sources of different kinds. Figures 4 and 5 show examples of them, with emission shown at 610 and $325 \mathrm{MHz}$. In Fig. 4, left panel, we display the source IRAS $20286+4105$, a star forming region (see also Ramachandran et al. 2017). Figure 4, right panel depicts for the first time at $325 \mathrm{MHz}$, a radio galaxy candidate, proposed as a counterpart of VHE sources (Martí et al. 2007). Figure 5 presents proplyd-like objects, probably photoionized by the brightest Cyg OB2 stars. In Figure 6, we show a small region of sky at $325 \mathrm{MHz}$ to illustrate the large number of radio sources with wide range of morphology.

\section{Radio observations of Galactic high-mass star forming regions}

GMRT is routinely used for probing the ionized emission associated with Galactic star forming regions. Das et al. (2018) have for the first time presented low-frequency high-resolution maps of two 
southern HII regions, G346.056-0.021 and G346.077-0.056 which are located at a distance of 10.9 kpc. The ionized emission mapped at 610 and $1280 \mathrm{MHz}$ are shown in Figure 7.
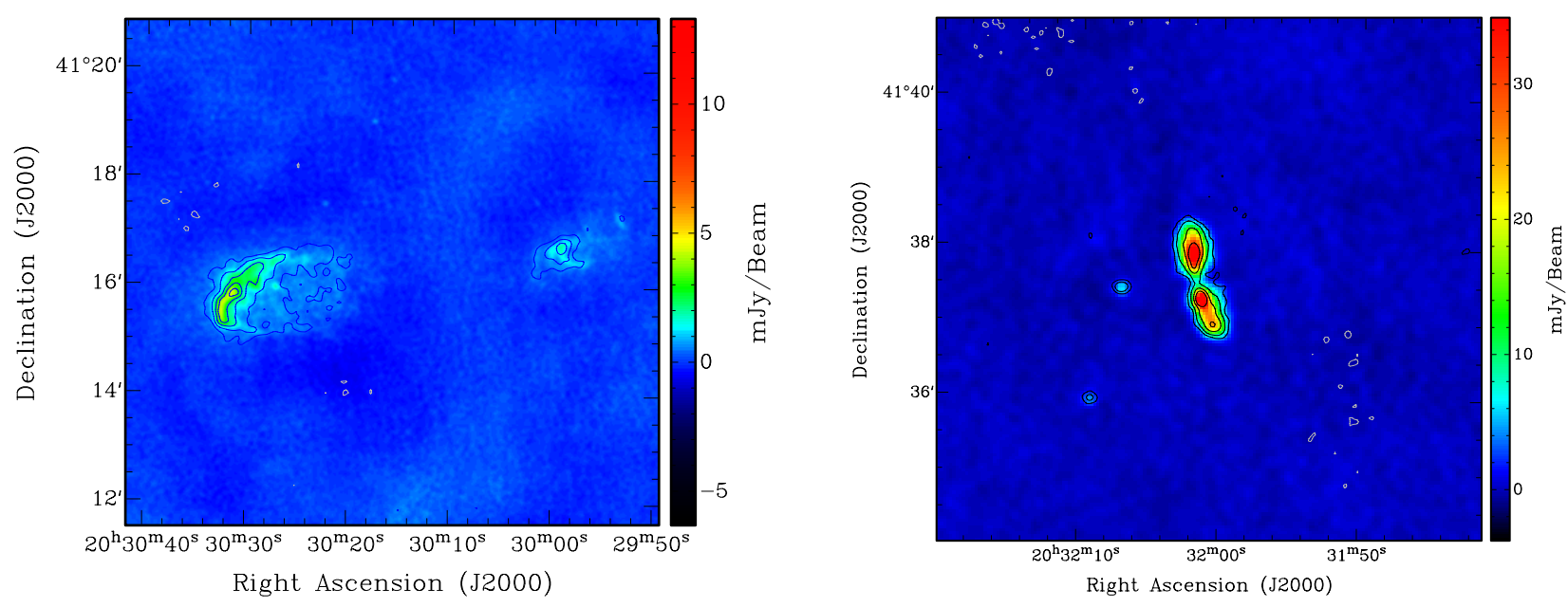

Figure 4: Left: Continuum emission towards the source IRAS $20286+4105$, at $610 \mathrm{MHz}$ (grey scale) and $325 \mathrm{MHz}$ (contours); contour levels: $\pm 1.5,3,5,7$ and $9 \mathrm{mJy}^{\text {beam }}{ }^{-1}$. Right: The proposed radio galaxy [PMI2007] J203201.7+413722 at 325MHz (grey scale) and $610 \mathrm{MHz}$ contours; contour levels are $\pm 0.3,1,3$ and $6 \mathrm{mJy}^{\text {beam }^{-1}}$.

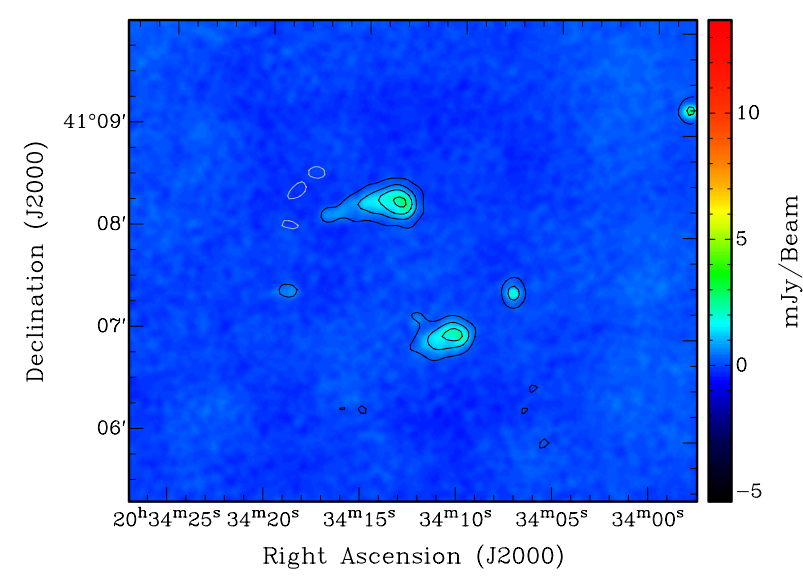

Figure 5: Protoplanetary-disk like sources near the center of the Cyg OB2 association, at 610 $\mathrm{MHz}$ (grey scale) and $325 \mathrm{MHz}$ (contours); contour levels are $\pm 1,3,5$ and $7 \mathrm{mJy}_{\text {beam }}{ }^{-1}$.

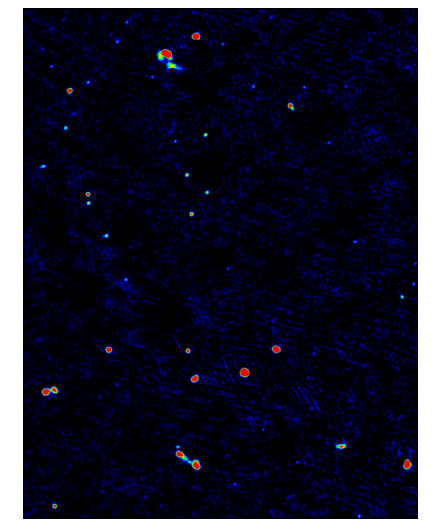

Figure 6: A small area $(30 \times 20$ arcmin $)$ from $325 \mathrm{MHz}$ survey, showing the variety of radio sources

The radio maps reveal the presence of diffuse ionized emission displaying distinct cometary morphologies. The $1280 \mathrm{MHz}$ flux densities translate to zero age main sequence spectral types in the range $\mathrm{O} 7.5 \mathrm{~V}-\mathrm{O} 7 \mathrm{~V}$ and $\mathrm{O} 8.5 \mathrm{~V}-\mathrm{O} 8 \mathrm{~V}$ for the ionizing stars of G346.056-0.021 and G346.077-0.056, respectively. Simple analytical calculations show that the bow-shock mechanism is less likely to be responsible for the observed cometary morphology. The variation of the ionized gas and the column density along the cometary axis favors the champagne-flow model for both HII regions. 


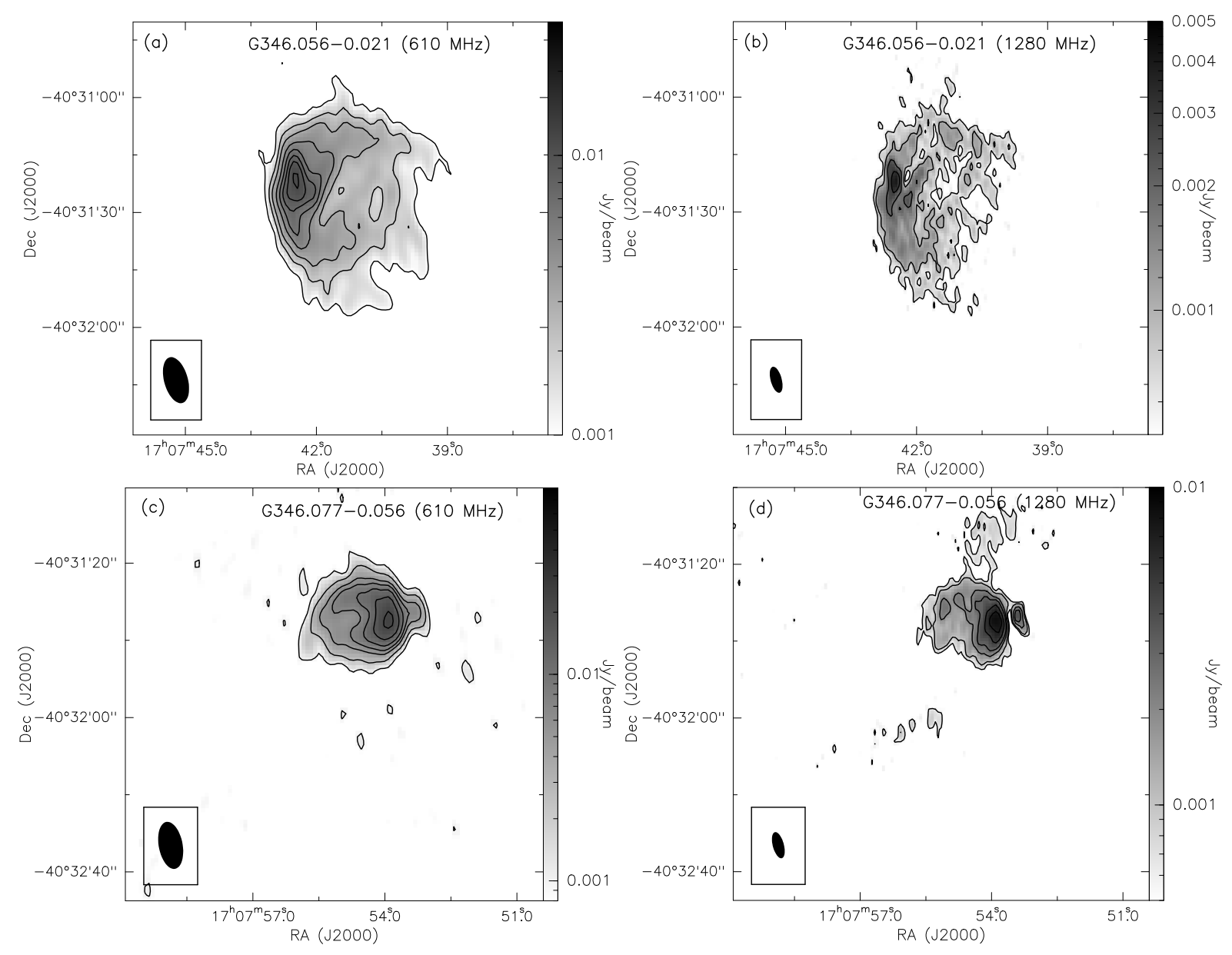

Figure 7: Ionized emission associated with the HII regions - (a) and (b) 610 and $1280 \mathrm{MHz}$ maps for the region associated with G346.056-0.021; (c) and (d) 610 and $1280 \mathrm{MHz}$ emission for the region around G346.077-0.056. The contour levels are 3, 5, 10, 15, 20, 25, 30, 35 times of $\sigma$ with $\sigma$ is $0.3 \mathrm{mJy} / \mathrm{beam}$ and $0.2 \mathrm{mJy} / \mathrm{beam}$ at $610 \mathrm{MHz}$ and $1280 \mathrm{MHz}$, respectively. Beam in each band is shown as filled ellipse. These maps are generated by setting the 'robustness parameter' to -5 and without any $u v$ tapering. This figure is reproduced from Das et al. (2018) with permission (C) ESO).

\section{Concluding remarks}

We have presented a preliminary overview of the deepest and wide area survey of the Cygnus OB2 region at $325 \mathrm{MHz}$ and $610 \mathrm{MHz}$ with the GMRT. The survey covers a wide area ( $~ 5$ square degrees) at sub-mJy sensitivity which helps to discover faint radio sources for the first time. The analysis of the full data set is still in progress.

The low radio frequency measured by the GMRT will preferentially pickup more non-thermal radio sources as compared to higher radio frequencies, considering the frequency dependence of synchrotron spectra, among which one finds potential counterparts for VHE sources. A good fraction of VHE sources do not have confirmed counterparts at any of the other branches of the electromagnetic spectrum, hence the so-called unidentified VHE source status. In the extra-galactic sky, most of the VHE sources with counterparts are blazars, a sub-class of AGN. However, VHE sources of Galactic origin are likely to belong to a wide range of categories, including supernovae remnants, pulsar wind nebulae, colliding wind binaries, microquasars, etc.

The deep investigation of the radio emission in a given region, in particular the Cygnus OB2 region very rich in various kinds of sources, constitutes the most obvious approach to investigate the 
origin of VHE sources in that region. The leptonic VHE emission and radio emission due to the synchrotron process do occur from the same population of relativistic electrons in several source types. Both radiations offer thus a complementary view of that non-thermal particle population. The detailed investigation of the sources detected in the field of our GMRT observations will allow for the determination of spectral indices, which is of first importance to characterize relativistic electron populations. On the other hand, accurate flux density measurements will provide important constraints on the energetics of the objects, and in particular on their non-thermal physics.

The catalogue of sources that is being built on the basis of this unprecedented data set will constitute the most abundant census of non-thermal sources in the Cygnus region to date.

\section{Acknowledgments}

We thank the staff of the GMRT that made these observations possible. GMRT is run by the National Centre for Radio Astrophysics of the Tata Institute of Fundamental Research. We also thank Huib Intema for extensive help in SPAM and scripts that were used for mosaicing the individual images. The authors thank the organizers of this fruitful meeting for the opportunity to present this oral contribution.

\section{References}

Bosch-Ramon V., Aharonian F. A., Paredes J. M. 2005, A\&A, 432, 609

Das S. R., Tej A., Vig S. et al. 2018, A\&A,612, A36

De Becker M., Raucq F. 2013, A\&A, 558, A28

De Becker M., Benaglia P., Romero G. E., Peri C. S. 2017, A\&A, 600, A47

Dubner G., Giacani E. 2015, A\&ARv, 23, 3

Intema H. T., van der Tol S., Coton W. D. et al. 2009, A\&A, 501, 1185

Knödlseder J. 2003, in Proceedings of A Massive Star Odyssey: From Main Sequence to Supernova, IAU Symp. 212, held in Lanzarote (Spain), June 2002

Mahy L., Rauw G., De Becker M. et al. 2013, A\&A, 550, 27

Marti J., Paredes J. M., Ishwara-CHandra C. H. et al. 2007, A\&A, 472, 557

Massaro F., D’Abrusco R., Giroletti M. et al. 2013, ApJS, 207, 4

Ramachandran V., Das S. R., Tej A. et al. 2017, MNRAS, 465, 4753

Reipurth B., Schneider N. 2008, Star Formation and Young Clusters in Cygnus, Handbook of Star Forming Regions, Volume I: The Northern Sky ASP Monograph Publications, Ed. B. Reipurth

Reynolds S. P., Gaensler B. M., Bocchino F. 2012, SSRv, 166, 231 